\title{
Functional neurological disorders in patients undergoing spinal surgery: illustrative case
}

\author{
*Ketan Yerneni, BA, Harsh Wadhwa, BS, Parastou Fatemi, MD, and Corinna C. Zygourakis, MD \\ Department of Neurological Surgery, Stanford University, Palo Alto, California
}

BACKGROUND "Conversion disorder" refers to bodily dysfunction characterized by either sensory or motor neurological symptoms that are unexplainable by a medical condition. Given their somatosensory context, such disorders often require extensive medical evaluation, and the diagnosis can only be made after structural disease is excluded or fails to account for the severity and/or spectrum of the patient's deficits.

OBSERVATIONS The authors briefly review functional psychiatric disorders and discuss the comprehensive workup of a patient with a functional postoperative neurological deficit, drawing from their recent experience with a patient who presented with conversion disorder immediately after undergoing anterior cervical discectomy and fusion.

LESSONS Conversion disorder has been found to be associated with bodily stress, requiring surgeons to be aware of this condition in the postoperative setting. This is especially true in neurosurgery, given the overlap of true neurological pathology, postoperative complications, and manifestations of conversion disorder. Although accurately diagnosing and managing patients with conversion disorder remains challenging, an understanding of the multifactorial nature of its etiology can help clinicians develop a methodical approach to this condition.

https://thejns.org/doi/abs/10.3171/CASE2068

KEYWORDS conversion disorder; functional neurological disorder; spine surgery

Conversion disorder (i.e., functional neurological symptom disorder [FNSD]) is a condition in which patients demonstrate neurological symptoms in the absence of structural disease, with symptoms ranging from limb weakness to sensorineural deficits and movement disorders. ${ }^{1}$ Patients with such symptoms constitute a not-insignificant portion of neurology patients, with certain groups reporting an incidence close to that of multiple sclerosis or Parkinson's disease. ${ }^{2} \mathrm{Al}-$ though functional disorders may remit spontaneously, these patients have consistently demonstrated poor long-term outcomes, with many remaining symptomatic or with disability up to 12 years after the initial diagnosis. ${ }^{3,4}$

Despite the clinical importance of conversion disorder, the mechanisms and dynamics behind this disease remain poorly understood relative to those of many other psychiatric and neurological conditions. Although conversion disorder has historically been believed to be, in part, a psychiatric response to a stressor, the Diagnostic and Statistical Manual of Mental Disorders, Fifth Edition (DSM-5) removed the association with conflicts or other stressors as an explicit diagnostic criterion, instead emphasizing the need to find positive clinical features such as Hoover's sign (which is defined in the context of our case presentation) in functional leg weakness.

More recently, preliminary magnetic resonance imaging (MRI) data suggested that some conversion disorder populations demonstrate structural brain alterations in the prefrontal and paralimbic regions. ${ }^{5} \mathrm{~A}$ recent diffusion tensor imaging study of patients with mixed symptoms demonstrated microstructural differences in the limbic and associative tracts as compared with those in healthy control subjects. ${ }^{6}$ Regardless of the mechanistic underpinnings, patients with conversion disorder must be accurately diagnosed because the disease may lead to unnecessary tests and healthcare costs and to irreversible health consequences via administration of inappropriate medical intervention.

Given the overlap of neurological symptoms in conversion disorder and perioperative neurosurgical complications, neurosurgeons need working knowledge of how to methodically discern the cause of

ABBREVIATIONS ACDF = anterior cervical discectomy and fusion; DSM-5 = Diagnostic and Statistical Manual of Mental Disorders, Fifth Edition; EEG = electroencephalography; FNSD = functional neurological symptom disorder; ICU = intensive care unit; MRI = magnetic resonance imaging; SSEP = somatosensory evoked potential.

INCLUDE WHEN CITING Published January 11, 2021; DOI: 10.3171/CASE2068.

SUBMITTED October 14, 2020. ACCEPTED October 30, 2020.

* K.Y. and H.W. contributed equally to this work.

(C) 2021 The authors, CC BY-NC-ND 4.0 (http://creativecommons.org/licenses/by-nc-nd/4.0/). 
symptomatology. Here, we report our recent experience with a patient who underwent anterior cervical discectomy and fusion (ACDF) and demonstrated no function in his legs immediately postoperatively. After undergoing a thorough workup of his symptoms, he was diagnosed with conversion disorder, or FNSD. We discuss the comprehensive workup of a postoperative neurological deficit, including when to consider conversion disorder and how to delineate this condition from other psychiatric illnesses.

\section{Illustrative Case}

\section{History and Presentation}

A 56-year-old man who was an active smoker with a history of head trauma, intermittent homelessness, drug abuse, schizoaffective disorder, major depressive episode with psychotic features, and suicidality presented to the clinic with worsening gait instability, bilateral worsening hand dexterity, and head and neck pain. His neurological examination was notable for $4 / 5$ strength in bilateral deltoids and biceps; 4-/5 strength in triceps, forearm extensors, and hand grip bilaterally; and $4 / 5$ strength in bilateral lower extremities. He reported numbness in both hands. His tandem gait was unsteady. His reflexes were 2+ and symmetric bilaterally, without clonus or Hoffman's sign. Cervical spine
MRI demonstrated severe central stenosis at C3-C4 with T2 cord signal change (Fig. 1A and B). Of note, the patient had previously been seen in a spine clinic, but attempts to perform cervical decompression surgery had been canceled. One was canceled because the patient tested positive for cocaine use, and another was canceled when the patient did not present for surgery. The patient was again offered C3-C4 ACDF, this time by a second neurosurgeon, and he provided consent.

\section{Operative and Postoperative Course}

A right-sided C3-C4 ACDF was performed in standard fashion with the patient under general anesthesia. No changes in baseline somatosensory evoked potentials (SSEPs) or motor evoked potentials were noted throughout the case, and the patient was extubated without complication. Immediately after surgery, the patient demonstrated spontaneous movement of his upper and lower extremities in the postoperative care unit. Soon after waking up, the patient endorsed improved strength in his upper extremities, including biceps, triceps, and handgrip, with no movement in his bilateral lower extremities and no sensation from his upper torso down to his legs. On examination, he had no voluntary movement in any muscle group in the lower extremities and no withdrawal to painful stimuli in the lower extremities.
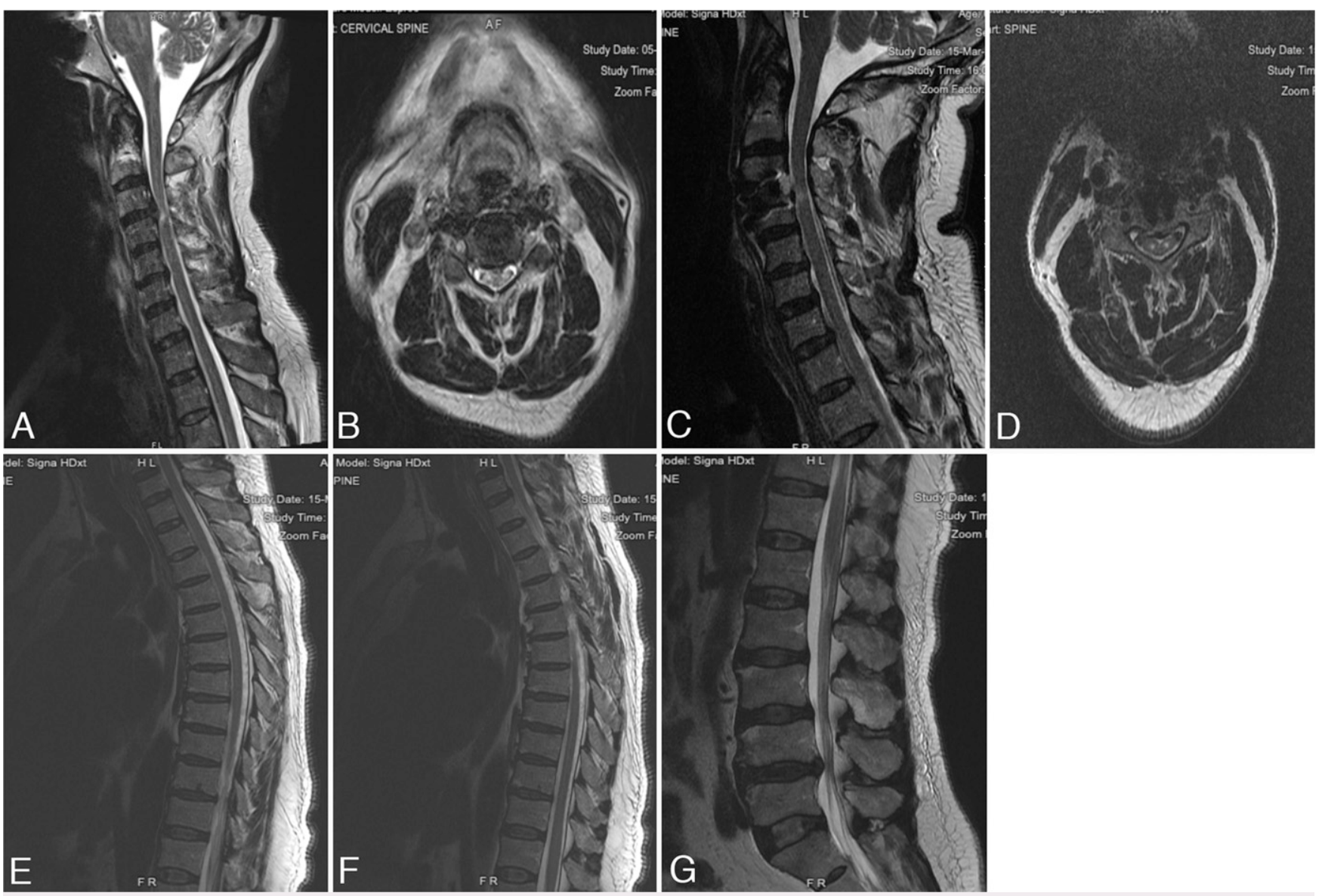

FIG. 1. A: Preoperative cervical spine MRI: sagittal T2. B: Preoperative cervical spine MRI: axial T2 at the C3-C4 level showing significant T2 signal cord change. C: Immediate postoperative cervical spine MRI: sagittal T2. D: Immediate postoperative cervical spine MRI: T2 axial at C3-C4 level showing stable T2 signal. E: Immediate postoperative thoracic spine MRI. F: Immediate postoperative thoracic spine MRI. G: Immediate postoperative lumbar spine MRI. 
Given the preserved motor and sensory examination findings in the upper extremities, as well as the flat, soft appearance of the wound and the lack of intraoperative monitoring changes, we investigated the cause of these symptoms with immediate imaging rather than returning to the operating room for wound exploration. Immediate MRI of the brain and the cervical, thoracic, and lumbar spine was performed and showed no acute pathology to explain his symptoms. The C3-C4 level was reasonably decompressed without evidence of epidural hematoma or other compression, with stable T2 signal cord change as compared with before surgery (Fig. 1C and D). There were no compressive lesions in the thoracic or lumbar spine to explain his lower extremity paresis (Fig. 1E-G). The findings of MRI of the brain, including a stroke protocol, were negative. The patient was transferred to the intensive care unit (ICU) for close neurological monitoring with hourly neurological checks, steroids, and elevated mean arterial blood pressures.

The patient continued to state that he was unable to move his legs when examined by the medical team and did not withdraw his legs even to painful stimulation. Overnight on postoperative day 1, ICU nursing staff reported that the patient was able to move his legs intermittently and found that his legs were in different positions when they exited and reentered his room. As a result, we stopped steroids and pressors and transferred the patient to the floor on the morning of postoperative day 1. On postoperative day 2 , the patient was able to consistently move his toes; when provided with distraction, he was able to move the remainder of his leg muscles as well. The result of his Hoover's test was positive bilaterally. Hoover's sign is the extension of the contralateral hip when the person is asked to lift their leg (even if they will not move that leg to command). Additionally, the patient intermittently reported other neurological ailments, including diplopia that was worse with central gaze in the absence of disconjugate eye movements.

We consulted the neurology service, which performed SSEP monitoring to ensure the integrity of the sensory pathways. The patient's SSEPs were found to be normal and consistent with those obtained intraoperatively. On postoperative day 3 , a member of the neurosurgical team observed the patient standing unassisted in front of his wheelchair while shaving in front of the mirror in his hospital room. Video electroencephalography (EEG) monitoring was subsequently performed in the patient's hospital room, showing the patient not only able to move his legs but also standing up independently. There was no noted seizure activity.

Throughout his hospital stay, the patient was verbally abusive to nursing and medical staff, frequently ordering interns/residents out of his room as he stated that he did not want to be taken care of by "child doctors." He often refused to cooperate with neurological examinations, and he would frequently change the topic. For example, a neurosurgery resident asked, "Mr. M, can you move your legs for me?" Patient M replied, "Why can't you get me some sunflower seeds? I need sunflower seeds right now." Patient advocates from the hospital met repeatedly with the patient and his daughter.

The neurosurgery and neurology teams reviewed the imaging and physical examination findings with the patient to reassure him of his lack of structural abnormalities. At this time, the patient reported that his legs would work fine intermittently, and he was in fact able to stand up at times. He was uncertain why he could not do so at the time of examination by the medical team.

Given the patient's inconsistent neurological examination findings, we consulted the psychiatry service, which believed his weakness to be a functional neurological disorder. The psychiatry team was unable to formally diagnose a factitious versus conversion disorder because it was unclear whether the patient had any motives for secondary gain, such as a desire for disability money, legal action, or additional pain medication prescriptions.

The physical therapists worked closely with the patient and recommended discharge to an acute inpatient rehabilitation facility. All facilities rejected admittance because of the patient's pattern of behavior. Furthermore, the patient repeatedly refused to go to an acute rehabilitation facility. The patient was ultimately discharged to home on postoperative day 5 . He was not participatory with strength examination at the time of discharge.

At his 1-month follow-up visit, the patient arrived in a wheelchair with a soft collar in place. Of note, the patient was not prescribed a collar during his hospital stay. He was able to ambulate independently when encouraged, and his strength was $4+/ 5$ in the upper extremities and $5 / 5$ in the lower extremities. The patient himself noted that the strength and sensation in his hands was improved compared with before surgery. He asked for a new collar and requested additional oxycodone prescriptions, becoming extremely agitated and upset when the surgeon explained to him that he was not eligible for additional opioid refills and requesting to speak with hospital management.

At his 1-year follow-up visit, the patient had stable numbness in his upper and lower extremities. He reported difficulty remembering things, sharp pain in the suboccipital area, and multiple recent falls. His neurological examination findings were stable. Cervical spine MRI did not reveal any significant stenosis. We recommended continued physical therapy and pain management of his chronic pain.

\section{Discussion}

\section{Observations}

Definition and Clinical Characteristics of Functional

Psychiatric Disorders

This case demonstrates the patient behavior, extensive physical examinations and diagnostic tests, and changing neurological symptoms without any physiological cause consistent with conversion disorder. Conversion disorder can be diagnosed by neurological symptoms that are inconsistent across portions of the examination or not associated with disease. ${ }^{7}$ Our patient also demonstrated the challenges of evaluating and treating a patient with acute neurological symptoms after spine surgery with no apparent pathological basis. However, similar psychiatric diagnoses such as factitious disorder or malingering cannot be ruled out. Because these psychiatric diseases are not well known to most practicing spine surgeons, we first clearly define them and later discuss the management techniques that are needed for these entities.

The DSM-5 diagnosis of conversion disorder requires each of the following: (1) one or more symptoms of altered voluntary motor or sensory function are present; (2) clinical findings demonstrate incongruity between neurological deficits and known patterns of disease; (3) the symptoms are not better explained by another mental or medical disorder; and (4) the symptoms cause significant distress or impairment or require medical evaluation. ${ }^{8}$ Generally speaking, and in contrast to patients with factitious disorder and malingering disorders, patients with conversion disorder are not intentionally feigning their symptoms and do not have external motivation (e.g., money, disability, opioid prescription) for the symptom presentation.

Factitious disorder is the deliberate feigning of symptoms in order to assume the sick-patient role. The patient with factitious disorder is intentionally producing their symptoms, without a clear external motivation, such as money or disability benefits. 
Malingering disorder is the deliberate feigning of symptoms for an explicit external purpose, such as to obtain money, disability benefits, opioid prescriptions, or other benefits (Fig. 2).

Although the external manifestations of these disorders may be similar, the underlying psychological needs and intrinsic driving factors behind these diseases are diverse. We do not know the exact reasons for our patient's behavior because he may have had a combination of conversion disorder with or without factitious or malingering characteristics. Our patient demonstrated several red flags that may alert a physician to a diagnosis of fabricated illness (Table 1). More specifically, our patient had a baseline mental and substance abuse disorder, was socioeconomically disadvantaged, and was intermittently homeless. In addition, he sought care from several neurosurgeons before undergoing surgery, had inconsistent examination findings with different providers, demonstrated an illness course that was not consistent with a C3-C4 injury level, had a history of prolonged opioid use, was noncompliant and disruptive on the unit, and demonstrated worsening behavior prior to discharge. Overall, his behavior led to multiple unnecessary diagnostic tests and treatments, including an ICU stay, initial treatment with steroids and blood pressure augmentation (that was stopped on postoperative day 1 when his inconsistent examination finding was noted), video EEG monitoring, SSEP testing, and repeated neurological and psychiatric consultations. In his follow-up visits, there was a suggestion of external motivation, namely threatened legal action and a request for further opioid prescriptions, although the patient did not follow through on these.

\section{Lessons}

Diagnosis of Functional Psychiatric Disorders in the Spine Patient

This case illustrates the importance of a detailed history and physical examination, along with comprehensive imaging, diagnostic testing, and appropriate consultations, in order to arrive at a functional neurological diagnosis, as this is a diagnosis of exclusion. Our patient underwent an uneventful ACDF, and his subsequent postoperative paresis was evaluated with immediate imaging of the neuroaxis. At the time, our differential diagnosis included intracerebral stroke, spinal cord compression/stroke or spinal epidural hematoma, or seizure. Given that the C3-C4 level was reasonably decompressed without evidence of epidural hematoma or other abnormalities, with a stable T2 signal cord change as compared with before surgery, the patient was transferred to the ICU for surveillance.

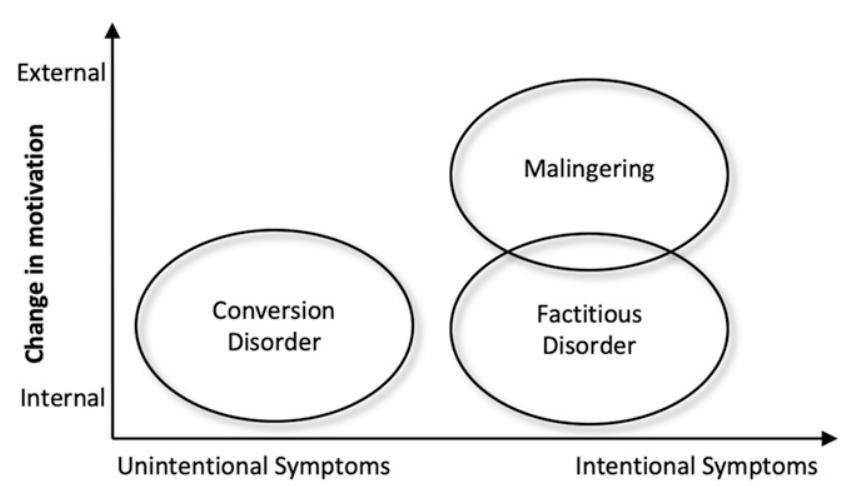

Symptom Production

FIG. 2. Model of functional neurological disease.

\section{TABLE 1. Clinical characteristics suggestive of fabricated illness}

The patient seeks treatment at various locations or with different providers.

The patient is an inconsistent, selective, or misleading informant.

The illness course is atypical and does not follow the natural presumed disease course.

Many tests, consultations, and medical/surgical treatments are performed, to no avail.

The magnitude of symptoms consistently exceeds objective pathology.

Some findings are self-induced or worsened through self-manipulation.

The patient eagerly agrees to or requests invasive medical therapy or surgery.

The patient predicts deteriorations, or there are exacerbations shortly before discharge.

At least one health care professional considers the diagnosis of factitious disorder.

The patient is noncompliant with treatment and disruptive on the unit. Laboratory/test results dispute information provided by patient.

The patient has a history of work in the healthcare field.

The patient engages in gratuitous, self-aggrandizing lying.

The patient has been prescribed opioid drugs.

The patient opposes psychiatric assessment.

Adapted from Bass and Halligan. ${ }^{18}$

One could argue that, in retrospect, an ICU stay was not needed for this patient. However, at the time that the patient was placed in the ICU, he had no movement in his lower extremities, and an early spinal stroke (not yet evident on imaging) or other occult neurological disease could not be definitively ruled out. It should also be noted that it was only in the ICU that the discrepancies in the patient's neurological status were first noted, putting psychiatric disorders on our differential, including somatic symptom disorder, depersonalization disorder, factitious disorder, conversion disorder, and malingering. We stress that clinicians faced with symptoms that are challenging to interpret should be cautious when diagnosing conversion disorder, regardless of how implicating the psychiatric history may be. Symptoms of an occult disease may be noticed by the patient at an early stage when physical examination findings or laboratory abnormalities may not be apparent to the clinician. ${ }^{9}$ Furthermore, certain rare diseases, including autoimmune limbic encephalitis or stiff person syndrome, may present with highly unexpected neurological findings. Additionally, worried patients may exaggerate their symptoms to convince a physician about their problem. ${ }^{10}$

In conjunction with various reports of the patient being able to use his lower extremities (confirmed by video EEG monitoring), as well as normal SSEPs and both neurology and psychiatry consults, our team verified that the patient had a functional deficit. This is one of the key diagnostic findings in conversion disorder: a deficit that is inconsistent at different times of observation. ${ }^{11}$ Although some may argue that this extensive workup (including ICU stay, multiple consults, SSEPs, and video EEG) was costly and unwarranted, our team believed that documentation of neurological pathway integrity with SSEPs and video confirmation of movement based on video EEG would be helpful in conversations with the patient and his family to explain his functional disease. The inclusion of the neurology, psychiatry, and physical

4 | J Neurosurg Case Lessons | Vol 1 | Issue 2 | January 11, 2021 
therapy services was instrumental not only in our understanding of the patient's symptoms but also for patient reassurance, which is an important aspect of the management and treatment of functional neurological disorders.

Previous cases of conversion paralysis after spine surgery have been reported in the literature. ${ }^{12-14}$ These patients typically present with neurological deficits in anatomical distributions inconsistent with disease. Assessment of such patients with reported motor deficits can be performed via the Hoover's sign test or the Spinal Injuries Center test. ${ }^{15}$ Generally, stroke and spinal disorders including cervical myelopathy or lumbar nerve root entrapment may cause weakness or sensory disturbance consistent with that seen in conversion disorder; however, patients with conversion disorder will have inconsistent limb weakness or movements. Figure 3 shows our proposed algorithm to aid spine surgeons in identifying possible functional neurological disorder in a postoperative patient with unexpected neurological symptoms.

Management of Functional Psychiatric Disorders in the Spine Patient

The management of conversion disorder and other functional neurological diseases remains difficult. The current first-line treatment involves educating the patient about their diagnosis. A prospective study of 54 patients with psychogenic nonepileptic seizures found that after the diagnosis was explained, $44 \%$ of patients immediately recovered. ${ }^{16}$ Some patients did experience a recurrence, however.
Educating patients about the diagnosis serves as the foundation on which any further treatment can be built. However, it is necessary for patients to believe the diagnosis, or they are unlikely to cooperate with additional treatment. Given the overlap of conversion disorder with various other psychiatric disorders, managing patients with a multidisciplinary team, including medical and psychological support, is essential. ${ }^{17}$ It is also useful to involve case management and/or a member of the hospital's legal team.

A few techniques for constructive confrontation of patients with functional neurological disorders include (1) "collecting firm evidence of fabrication" (e.g., in our case, normal MRI, SSEPs, and video EEG evidence of leg movement); (2) "discussing the case with a psychiatrist (or a member of the hospital's legal team if no psychiatrist is available)"; (3) "arranging a meeting to collate the facts, devise a strategy, and discuss with their primary care doctor"; (4) "confronting the patient in a non-judgmental and non-punitive way, with a proposal of support and ongoing follow-up"; and (5) "documenting a full record of the meeting and its outcome in the patient record."18

In summary, functional neurological disorders such as conversion disorder, factitious disorder, or malingering may present as new neurological deficits after spine surgery. It is important for spine surgeons to have a comprehensive algorithm for working up patients with new neurological deficits after spine surgery and to have an understanding of the various types of psychiatric disorders that can

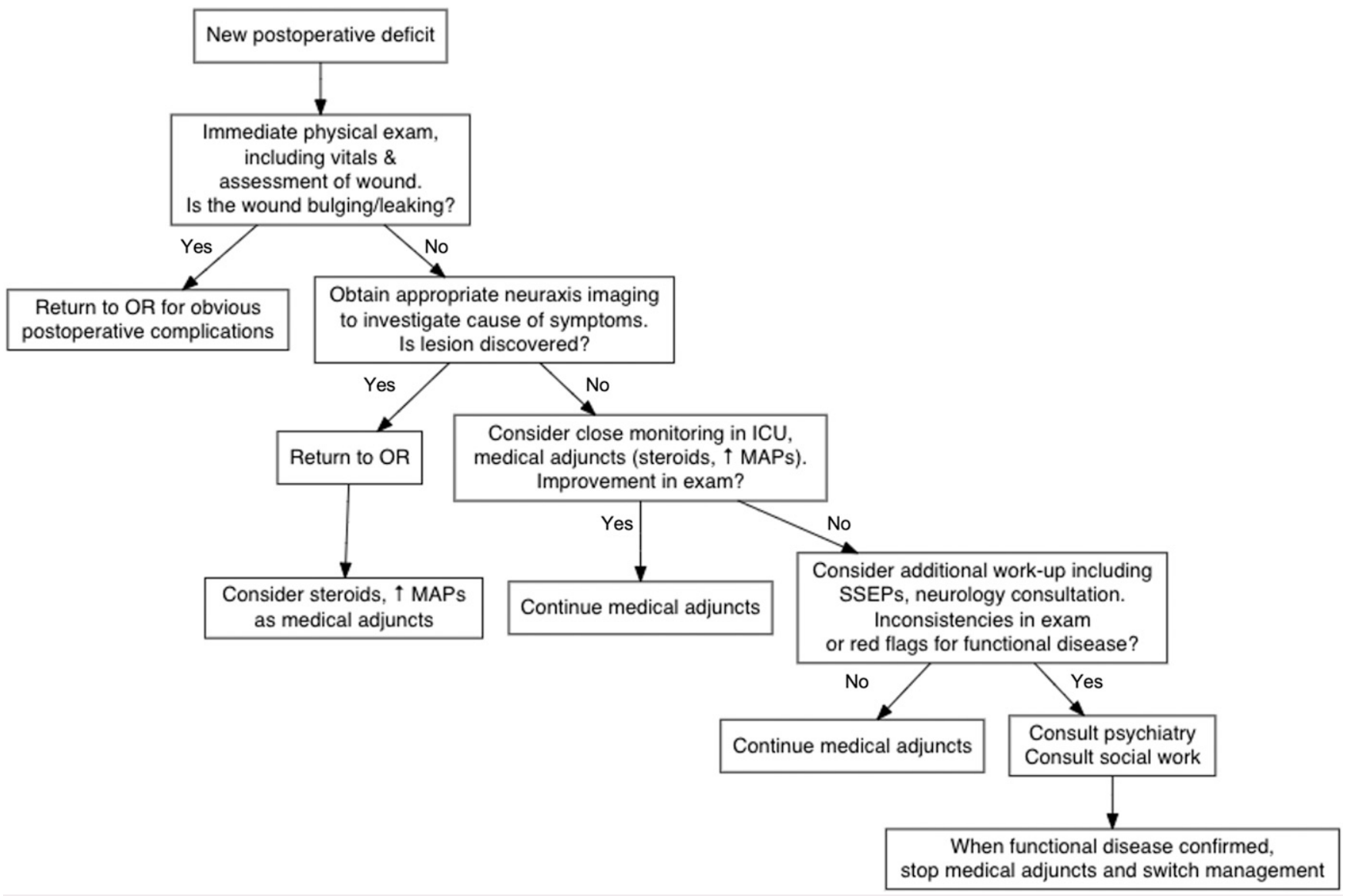

FIG. 3. Flowchart for workup and management of new postoperative neurological deficit in a spine patient. MAP = mean arterial pressure; OR = operating room. 
impact their patients. These functional neurological disorders have varying degrees of internal versus external motivation, as well as unintentional versus intentional production of symptoms. As a result, they can be very challenging to manage, and surgeons need a different skill set and a multidisciplinary team to appropriately care for these patients.

\section{References}

1. Ludwig L, Pasman JA, Nicholson T, et al. Stressful life events and maltreatment in conversion (functional neurological) disorder: systematic review and meta-analysis of case-control studies. Lancet Psychiatry. 2018;5(4):307-320.

2. Stone J, Carson A, Duncan R, et al. Symptoms 'unexplained by organic disease' in 1144 new neurology out-patients: how often does the diagnosis change at follow-up? Brain. 2009;132(Pt 10): 2878-2888.

3. Stone J, Sharpe M, Rothwell PM, et al. The 12 year prognosis of unilateral functional weakness and sensory disturbance. J Neurol Neurosurg Psychiatry. 2003;74(5):591-596.

4. Reuber M, Pukrop R, Bauer J, et al. Outcome in psychogenic nonepileptic seizures: 1 to 10-year follow-up in 164 patients. Ann Neurol. 2003;53(3):305-311.

5. Bègue I, Adams $C$, Stone J, et al. Structural alterations in functional neurological disorder and related conditions: a software and hardware problem? Neuroimage Clin. 2019;22:101798.

6. Diez I, Williams B, Kubicki MR, et al. Reduced limbic microstructural integrity in functional neurological disorder. Psychol Med. 2019. In press.

7. O'Neal MA, Baslet G. Treatment for patients with a functional neurological disorder (conversion disorder): an integrated approach. Am J Psychiatry. 2018;175(4):307-314.

8. American Psychiatric Association. Diagnostic and Statistical Manual of Mental Disorders: DSM-5. 5th ed. American Psychiatric Association; 2013.

9. Stone J, Reuber M, Carson A. Functional symptoms in neurology: mimics and chameleons. Pract Neurol. 2013;13(2):104-113.

10. Yetzy SH, Parish BS. Somatoform disorders. In: Tasman A, Kay J Lieberman JA, et al, eds. Psychiatry. 3rd ed. John Wiley \& Sons; 2008:1510-1547.

11. Stone J, Warlow $C$, Sharpe M. The symptom of functional weakness a controlled study of 107 patients. Brain. 2010;133(Pt 5):1537-1551.
12. Boudissa M, Castelain JE, Boissière L, et al. Conversion paralysis after cervical spine arthroplasty: a case report and literature review. Orthop Traumatol Surg Res. 2015;101(5):637-641.

13. Zhu L, Ni B, Guo Q. Hysterical paralysis after spinal surgery. Rheumatol Int. 2012;32(12):4077-4078.

14. Hsieh MK, Chang CN, Hsiao MC, et al. Conversion paralysis after surgery for lumbar disc herniation. Spine (Phila Pa 1976). 2010 35(8):E308-E310.

15. Yugué I, Shiba K, Ueta T, et al. A new clinical evaluation for hysterical paralysis. Spine (Phila Pa 1976). 2004;29(17): 1910-1913.

16. Duncan R, Razvi S, Mulhern S. Newly presenting psychogenic nonepileptic seizures: incidence, population characteristics, and early outcome from a prospective audit of a first seizure clinic. Epilepsy Behav. 2011;20(2):308-311.

17. Mohandas P, Bewley A, Taylor R. Dermatitis artefacta and artefactual skin disease: the need for a psychodermatology multidisciplinary team to treat a difficult condition. $\mathrm{Br} J$ Dermatol. 2013;169(3):600-606.

18. Bass $C$, Halligan P. Factitious disorders and malingering: challenges for clinical assessment and management. Lancet. 2014; 383(9926):1422-1432.

\section{Disclosures}

Dr. Zygourakis is a consultant to Stryker, Globus, and Spine Align and has received a grant from Stryker for work unrelated to this study.

\section{Author Contributions}

Conception and design: Zygourakis, Yerneni. Acquisition of data: all authors. Analysis and interpretation of data: all authors. Drafting the article: all authors. Critically revising the article: all authors. Reviewed submitted version of manuscript: Zygourakis, Yerneni, Wadhwa. Approved the final version of the manuscript on behalf of all authors: Zygourakis. Administrative/technical/material support: Zygourakis, Yerneni. Study supervision: Zygourakis.

\section{Correspondence}

Corinna C. Zygourakis: Stanford University School of Medicine, Palo Alto, CA. corinnaz@stanford.edu. 